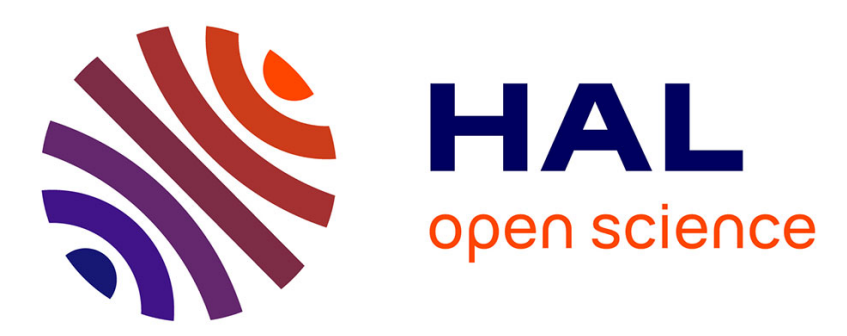

\title{
Résistance à la propagation d'un composite à fibres $\mathrm{C}-\mathrm{SiC}$
}

\author{
M. Gomina, P. Fourvel, F. Osterstock, J.L. Chermant
}

\section{To cite this version:}

M. Gomina, P. Fourvel, F. Osterstock, J.L. Chermant. Résistance à la propagation d'un composite à fibres C-SiC. Revue de Physique Appliquée, 1988, 23 (3), pp.183-191. 10.1051/rphysap:01988002303018300 . jpa-00245761

\section{HAL Id: jpa-00245761 https://hal.science/jpa-00245761}

Submitted on 1 Jan 1988

HAL is a multi-disciplinary open access archive for the deposit and dissemination of scientific research documents, whether they are published or not. The documents may come from teaching and research institutions in France or abroad, or from public or private research centers.
L'archive ouverte pluridisciplinaire HAL, est destinée au dépôt et à la diffusion de documents scientifiques de niveau recherche, publiés ou non, émanant des établissements d'enseignement et de recherche français ou étrangers, des laboratoires publics ou privés. 


\title{
Résistance à la propagation d'un composite à fibres $\mathrm{C}-\mathrm{SiC}$
}

\author{
M. Gomina, P. Fourvel, F. Osterstock et J. L. Chermant \\ LERMAT, ISMRa-Université de Caen, Boulevard du Maréchal-Juin, 14032 Caen Cedex, France
}

(Reçu le 21 août 1987, révisé le 3 décembre 1987, accepté le 16 décembre 1987)

\begin{abstract}
Résumé. - Dans cet article nous présentons des courbes de résistance à la propagation de fissure de matériaux composites céramiques à fibres, C-SiC. Ces courbes ont été obtenues par la méthode de Garwood et de Garwood corrigée des déformations résiduelles et par la méthode basée sur l'incrément de travail de rupture (méthode de Sakai). Le bon accord entre les longueurs des fissures mesurées par une méthode visuelle et celles calculées par une méthode itérative nous a conduit à utiliser ces dernières pour évaluer la résistance à la propagation de fissure. Les résultats conduisent à une même relation linéaire entre les paramètres estimés par les méthodes de Garwood et leurs homologues estimés par la méthode de Sakai.
\end{abstract}

\begin{abstract}
In this paper we present crack propagation resistance curves obtained from C-SiC fiber ceramic composites. These curves were derived by the Garwood method and the Garwood method with corrections to the residual strains, and by the method based on the incremental work of fracture (Sakai method). The good agreement between the optically measured crack lengths and those calculated from an iterative method led to use this last method for the evaluation of the crack resistance. The results indicate a unique linear relationship between the parameters estimated by the Garwood methods and their homologuous estimated from the Sakai method.
\end{abstract}

\section{Introduction.}

L'étude du comportement mécanique des matériaux repose sur la connaissance des réponses mécaniques aux sollicitations extérieures. Ainsi, la Mécanique Linéaire de la Rupture (MLR) permet l'évaluation de la résistance à la propagation de fissure, à partir de la connaissance de l'énergie mécanique fournie au matériau pour le porter à un niveau d'endommagement donné. Le paramètre d'endommagement relève des effets d'accommodation dans le volume du matériau contraint et il n'existe pas encore de paramètre unique fiable pour le caractériser : certains utilisent la variation de compliance, alors que d'autres définissent l'endommagement par la densité de microfissures créées $[1,2]$.

Généralement, l'endommagement est directement estimé à l'aide de la longueur de fissure dans le matériau. La méthode du marquage à l'encre [3-5] donne de bons résultats, mais elle s'applique difficilement en cours d'essai, bien que l'utilisation de laques de différentes couleurs soit envisageable. Pour les matériaux à structure fine, la longueur de fissure est calculée à partir des valeurs de compliance à l'aide des équations de calibrations usuelles [6]. Mais pour la plupart des matériaux ces équations ne s'appliquent pas et il faut construire une courbe de calibration de compliance pour chaque type de matériau. La méthode la plus immédiate consiste à utiliser des éprouvettes de dimensions identiques comportant des longueurs d'entaille différentes; mais il a été observé que pour un matériau donné, la réponse mécanique est fonction de la finesse du rayon en fond d'entaille $[7,8]$. Pour les métaux la courbe de calibration de compliance peut être obtenue à partir de fissures de fatigue [9]. Les longueurs de fissure en cours de rupture sont ensuite estimées par une méthode ultrasonore $[10,11]$ ou par les méthodes de mesure de tension électrique sur l'éprouvette rendue conductrice ou portant un réseau de grilles résistives $[12,13]$.

L'observation directe de l'avancée de la fissure au cours de l'essai de rupture semble, à l'heure actuelle, être la meilleure méthode de mesure. Elle a été pratiquée, par l'équipe du Dr R. F. Pabst de l'Institut Max Planck de Stuttgart, à l'ambiante et à haute température en donnant d'excellents résultats [14].

On se propose ici d'utiliser cette méthode pour 
mesurer les longueurs de fissure au cours d'un essai de rupture par charge-décharge sur un composite à fibres C-SiC. Ces valeurs seront comparées à celles obtenues à partir de la mesure de la compliance sur les cycles de charge-décharge et calculées d'après l'expression itérative de $\mathrm{H}$. Tada et al. [15].

Le but recherché est de valider cette expression pour les composites C-SiC et de l'utiliser pour l'exploitation des courbes force-déplacement. Ceci devra permettre de comparer deux approches thermodynamiques de la rupture : celle de l'intégrale $J$ selon la méthode proposée par Garwood et al. [16], avec et sans la correction de la déformation plastique d'une part, et celle par la méthode de Sakai et al. [17] d'autre part.

Ces essais, réalisés sur des éprouvettes de flexion trois points seront complétés par ceux sur des éprouvettes de traction compacte afin d'observer l'effet du type d'essai.

\section{Matériaux et techniques de mesure.}

2.1 MATÉriaU. - Dans ces composites céramiques, le renfort est constitué de monofilaments de carbone de 3 à $4 \mathrm{~cm}$ de long $\left(\varnothing=8\right.$ à $10 \mu \mathrm{m} ; \sigma_{\mathrm{r}}=1500$ $\mathrm{MPa}$ ) obtenus à partir d'un précurseur en fibres synthétiques PAN. Ces monofilaments sont torsadés pour former des mèches qui seront tissées (satin de 5) en un motif bidirectionnel. Ce tissu est ensuite empilé sur une carcasse graphite et compressé pour donner un taux volumique de fibres de $28 \%$ pour le matériau fini. La matrice $\mathrm{SiC}$ est obtenue par la méthode CVI [18], ce qui explique que ses caractéristiques physiques soient mal connues. On estime le module d'élasticité $E_{\mathrm{m}}=350 \mathrm{GPa}$; le coefficient de Poisson $\nu_{\mathrm{m}}=0,2$; le taux critique d'énergie de rupture $G_{\mathrm{c}}=50$ à $60 \mathrm{Jm}^{2}$ et le coefficient de dilatation thermique $\alpha_{\mathrm{m}}=4,6 \times 10^{-6} \mathrm{~K}^{-1}$. Le matériau fini (Fig. 1) présente une porosité $V_{\mathrm{p}} \simeq 15$ à $20 \%$ et

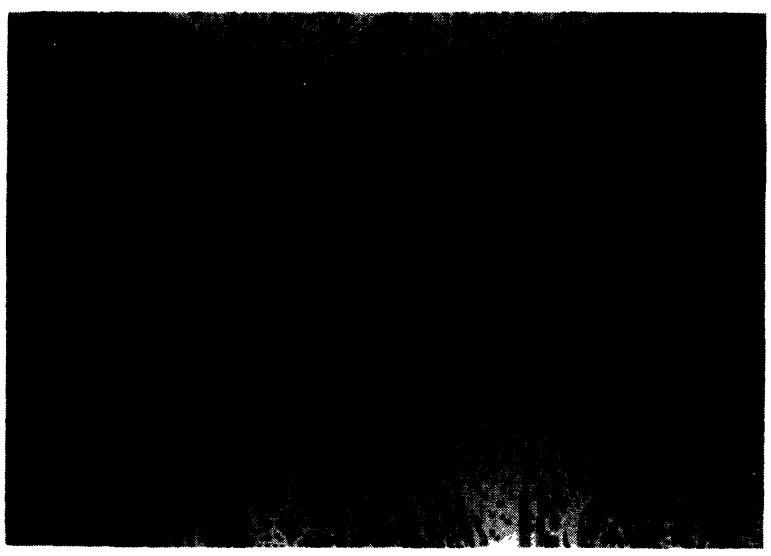

Fig. 1. - Micrographie d'un échantillon en C-SiC (provenance SEP).

[An optical micrography of a C-SiC composite material (from S.E.P., Bordeaux).] une densité de 2,2 à 2,5. La répartition en taille des pores et la distribution radiale des fibres dans les torons ont fait l'objet d'une étude morphologique particulière [19].

2.2 TeChnique de mesure. - Les essais de rupture ont été conduits sur des éprouvettes de flexion 3 points (SENB) et sur des éprouvettes de traction compacte (CT) (essais réalisés à la SEP-Bordeaux pour ces dernières) d'orientation « sur chant " notée $(1,2)$ (Fig. 2). Les dimensions des éprouvettes testées sont données dans le tableau I.

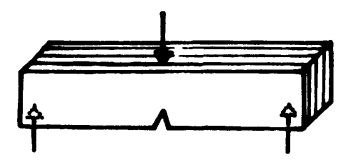

orient ation (1,2)

Fig. 2. - Orientations relatives du plan des strates de tissu et de la direction de l'effort de traction (pour une éprouvette de flexion 3 points).

[Relative orientations of the laminates plane and the applied tensile stress direction (in three point bending tests).]

Les essais de chargement ont été réalisés sur une machine de traction Schenck (pour les deux éprouvettes $100 \times 10 \times 5,5 \mathrm{~mm}^{3}$ entaillées à $a_{0} / W=0,35$ et 0,41 ) ou sur une machine Instron modèle 1185 en adoptant une vitesse de chargement de $20 \mu \mathrm{m} / \mathrm{min}$. Pour les éprouvettes de flexion, la déflexion est mesurée sous l'éprouvette à l'aide d'un palpeur inductif et pour les éprouvettes de traction compacte le déplacement du point d'application de la charge est mesurée à l'aide d'un extensomètre Instron calibré pour $1 \mathrm{~mm}=10 \mathrm{~V}$ DC. Les distances entre appuis étaient de $40 \mathrm{~mm}$ et $60 \mathrm{~mm}$ respectivement pour les éprouvettes SENB de dimensions $50 \times$ $10 \times 5 \mathrm{~mm}^{3}$ et $70 \times 15 \times 7,5 \mathrm{~mm}^{3}$; et de $90 \mathrm{~mm}$ pour toutes les autres éprouvettes SENB.

Une chaîne d'émission acoustique Dunegan 3000 a été utilisée avec un capteur piézoélectrique de bande passante $100-350 \mathrm{kHz}$, de gain $40 \mathrm{~dB}$ et le seuil fixé à $28 \mathrm{~dB}$ pour le suivi d'émission acoustique lors des essais de flexion, en utilisant le mode de comptage cumulé du nombre de coups.

Un microscope optique, mobile sur un axe vertical, permet de suivre l'avancée de la fissure sur la face polie de l'éprouvette au cours de l'essai de rupture. Le montage permet la visualisation de la surface polie de l'éprouvette sur un écran de télévision et l'enregistrement sur magnétoscope de l'avancée de la fissure au cours de l'essai de rupture, ainsi que des prises de vue à l'aide d'un appareil photo. La longueur de la fissure est mesurée d'après le signal d'un palpeur inductif solidaire du bâti portant le microscope optique. Le schéma de l'appareillage de mesure est présenté sur la figure 3. 
Tableau I. - Caractéristiques dimensionnelles et résultats des mesures d'énergie pour les éprouvettes $\mathrm{C}-\mathrm{SiC}$ testées. L est la distance entre appuis.

[Geometric dimensions and measured energy values for the specimens tested, $L$ is the span in three point bending tests.]

Méthode de Garwood et al. Méthode de Sakai et al.

\begin{tabular}{|c|c|c|c|c|c|c|c|}
\hline \multicolumn{2}{|l|}{ I. SENB } & \multicolumn{5}{|c|}{ a) $50 \times 10 \times 5 \mathrm{~mm}^{3} ; L=40 \mathrm{~mm}$} & \multirow[b]{2}{*}{$J^{S}\left(\mathrm{Jm}^{-2}\right)$} \\
\hline Réf. & $a(\mathrm{~mm})$ & $J^{\mathrm{G}}\left(\mathrm{J} / \mathrm{m}^{2}\right)$ & $J^{\mathrm{GC}}\left(\mathrm{J} / \mathrm{m}^{2}\right)$ & $d(\mathrm{~mm})$ & $a(\mathrm{~mm})$ & $R_{\mathrm{c}}\left(\mathrm{Jm}^{-2}\right)$ & \\
\hline F4 & 3,0 & 2260 & 1080 & 2,5 & 3,0 & 1600 & 700 \\
\hline F5 & 4,0 & 2440 & 1110 & 2,0 & 4,0 & 1800 & 810 \\
\hline F3.6 & 5,0 & 1440 & 730 & 2,0 & 5,0 & 1260 & 610 \\
\hline F3.7 & 5,4 & 2160 & 1000 & 2,1 & 5,4 & 1630 & 700 \\
\hline F3.8 & 5,7 & 2160 & 1040 & 1,6 & 5,7 & 1920 & 900 \\
\hline E3.17 & 6,0 & 1850 & 720 & 2,0 & 6,0 & 1200 & 450 \\
\hline E3.18 & 7,0 & 2540 & 1100 & 1,5 & 7,0 & 1400 & 860 \\
\hline \multicolumn{8}{|c|}{ b) $100 \times 18,7 \times 5,5 \mathrm{~mm}^{3} ; L=90 \mathrm{~mm}$} \\
\hline 11 & 5,6 & 7000 & 3000 & & 5,6 & 4930 & 2330 \\
\hline 10 & 7,4 & 6500 & 2700 & & 7,4 & 5070 & 2270 \\
\hline \multicolumn{8}{|c|}{ c) $100 \times 30 \times 5,5 \mathrm{~mm}^{3} ; L=90 \mathrm{~mm}$} \\
\hline 13 & $9,1$. & 6420 & 2750 & & 9,1 & 4600 & 2100 \\
\hline 12 & 12,0 & 5770 & 2550 & & 12,0 & 4250 & 2250 \\
\hline \multicolumn{8}{|c|}{ d) $100 \times 10 \times 5,5 \mathrm{~mm}^{3} ; L=90 \mathrm{~mm}$} \\
\hline 1 & 3,5 & 3200 & 1500 & 4,0 & 3,5 & 2680 & 920 \\
\hline 2 & 4,2 & 2600 & 1200 & 4,0 & 4,2 & $2500 / 3000$ & 900 \\
\hline \multicolumn{8}{|c|}{ e) $70 \times 15 \times 7,5 \mathrm{~mm}^{3} ; L=60 \mathrm{~mm}$} \\
\hline G4 & 7,5 & 3000 & 1000 & 2,4 & 7,5 & 2200 & 920 \\
\hline G5 & 9,0 & 3100 & 1400 & 2,0 & 9,0 & 1970 & 870 \\
\hline \multicolumn{8}{|c|}{ II. CT $25 \times 24 \times 10 \mathrm{~mm}^{3}$} \\
\hline A & $\mathrm{a} / \mathrm{w}=0,5$ & 2200 & 900 & & 0,5 & 1620 & 750 \\
\hline B & 0,5 & 2300 & 880 & & 0,5 & 1700 & 770 \\
\hline D & 0,4 & 2300 & 870 & & 0,4 & 1600 & 800 \\
\hline
\end{tabular}

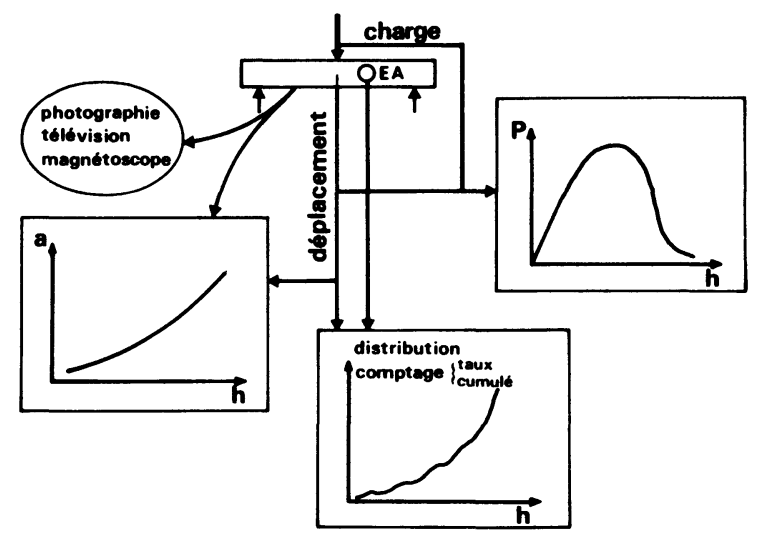

Fig. 3. - Schéma de l'appareillage utilisé pour les essais de flexion avec mesure directe de la longueur de fissure.

[Schematic of the apparatus used when optically measuring the crack length in a three point bending test.]
Parmi les méthodes proposées pour évaluer l'endommagement en dehors du cadre linéaire élastique de la rupture, nous avons retenu celle de l'intégrale $J$ et celle de Sakai [17].

2.2.1 Méthodes de Garwood. - Dans le cas des matériaux métalliques, où il apparaît une grande zone de déformation plastique en fond de fissure, Rice [20] a proposé un paramètre $J$ (intégrale de Rice) pour caractériser le champ de déformation en avant de la fissure. Ce paramètre d'énergie $J$ est une extension de la notion de résistance à la propagation, $R$, au cas d'un solide linéaire non élastique. Dans le cas général d'un solide non linéaire non élastique (matériau élastoplastique), Garwood et al. [16] ont proposé l'expression itérative (Fig. 4a) pour les essais de flexion :

$$
J_{n}^{\mathrm{G}}=J_{n-1}^{\mathrm{G}} \frac{W-a_{n}}{W-a_{n-1}}+2 \frac{U_{n}}{B\left(W-a_{n-1}\right)} .
$$




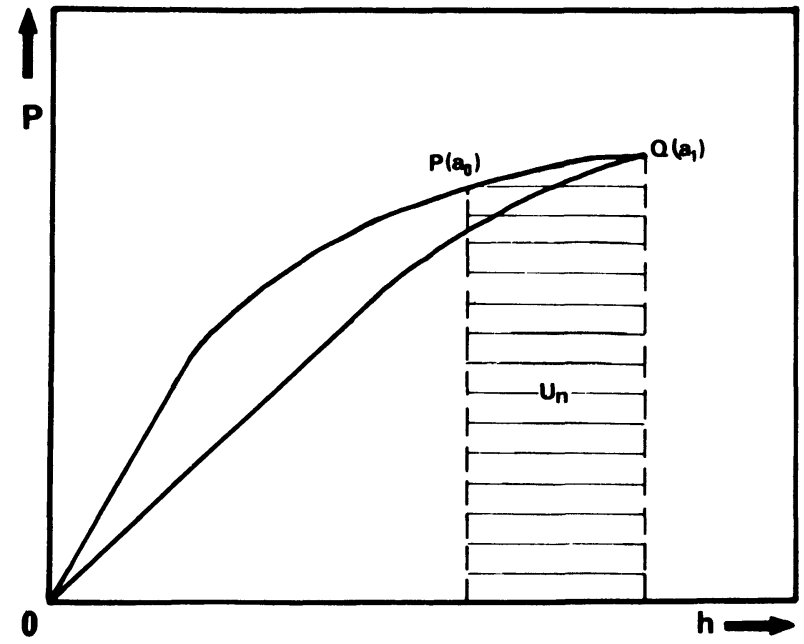

a)

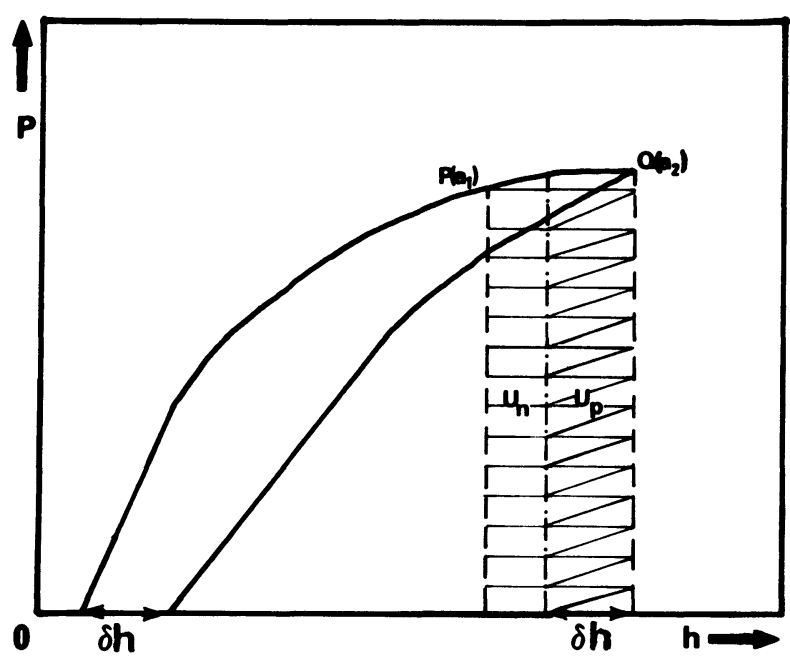

b)

Fig. 4. - Illustration de la méthode de Garwood : a) pour un solide non linéaire élastique ; b) pour un solide non linéaire non élastique, avec corrections dues aux déplacements résiduels.

[Schematic of the Garwood method : a) for a linear elastic body ; b) for a non-linear non-elastic body, with corrections to the residual displacements.]

Pour tenir compte des déformations résiduelles observées sur les courbes de chargement, l'approche de Garwood est corrigée, pour un déplacement permanent $\delta h$, par un terme d'énergie $U_{\mathrm{p}}$ correspondant au travail de la charge appliquée pour réaliser cette déformation. La correction apportée par Fantozzi et Bouaouadja [23] est schématisée sur la figure 4 , où l'accroissement de l'énergie mécanique $\left(U_{n}-U_{n-1}\right)$ entre les valeurs $a_{n}$ et $a_{n-1}$ est réduite par le terme «d'énergie plastique » $U_{p}$. Ce terme $U_{p}$ est une estimation de la contribution du déplacement permanent $\delta h$. Les valeurs $J$ ainsi mesurées sont notées $J^{\mathrm{Gc}}$ et dénommées «Valeurs de l'intégrale $J$ corrigées » et elles constituent les mesures de l'énergie de propagation de la fissure :

$$
J_{n}^{\mathrm{GC}}=J_{n-1}^{\mathrm{GC}} \frac{W-a_{n}}{W-a_{n-1}}+2 \frac{U_{n}-U_{p}}{B\left(W-a_{n-1}\right)}
$$

avec

$$
J_{0}=\frac{2 U_{0}}{B\left(W-a_{0}\right)}
$$

: $a_{n}$ les longueurs de fissure,

$U_{0}$ : énergie élastique de l'éprouvette pour l'entaille $a_{0}$,

$U_{n}$ : travail des forces extérieures entre $a_{n-1}$ les longueurs et $a_{n}$,

$U_{p}$ : énergie associée au déplacement résiduel entre les longueurs $a_{n}$ et $a_{n-1}$.

2.2.2 Méthode de Sakai. - Egalement pour tenir compte des déplacements résiduels qui apparaissent

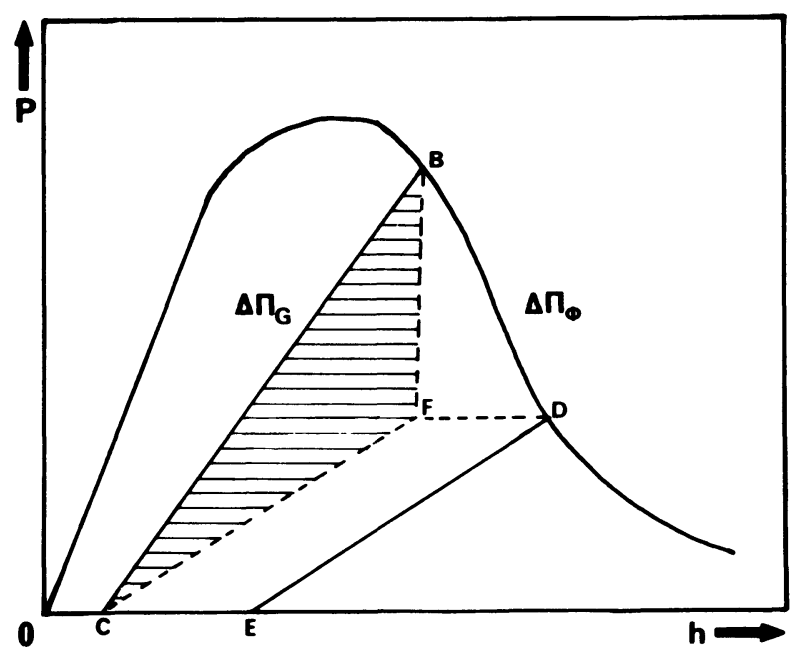

a)

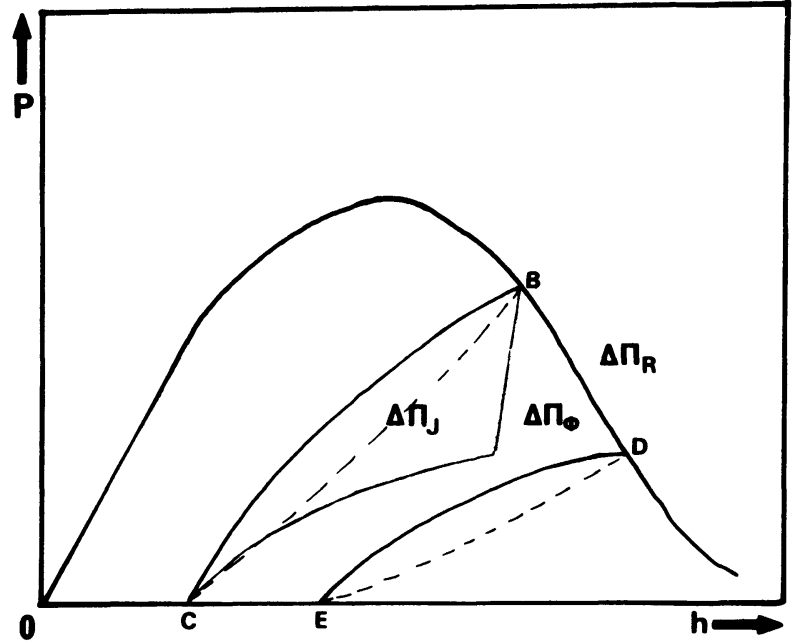

b)

Fig. 5. - Méthode de décomposition du travail incrémental de rupture : a) cas d'un solide linéaire non élastique ; b) cas d'un solide non linéaire non élastique.

[Schematic of the decomposition method applied to the incremental work of rupture : a) for a linear non-elastic body ; b) for a non-linear non-elastic body.] 
au cours des cyclages, Sakai et al. [17] décomposent la résistance totale à la propagation, $R$, en un terme lié à la variation de compliance, $\tilde{G}_{\mathrm{c}}$, et un terme $\phi$, fonction de la déformation résiduelle (Fig. 5a) soit

avec :

$$
R=\tilde{G}_{\mathrm{c}}+\phi
$$

$$
R=\frac{\Delta \pi_{R}}{\Delta A} ; \quad \tilde{G}_{\mathrm{c}}=\frac{\Delta \pi_{G}}{\Delta A} \quad \text { et } \quad \phi=\frac{\Delta \pi_{\phi}}{\Delta A} .
$$

Dans le cas où les trajets de charge-décharge ne sont pas linéaires, le terme $\tilde{G}_{\mathrm{c}}$ est remplacé par la quantité $J$ (Fig. 5b) avec :

$$
J=\frac{\Delta \pi_{J}}{\Delta A}
$$

\section{Résultats et discussion.}

La figure 6 a présente, pour un composite céramique $\mathrm{C}-\mathrm{SiC}$ d'orientation $(1,2)$, un enregistrement en cyclage de charge en fonction du déplacement du

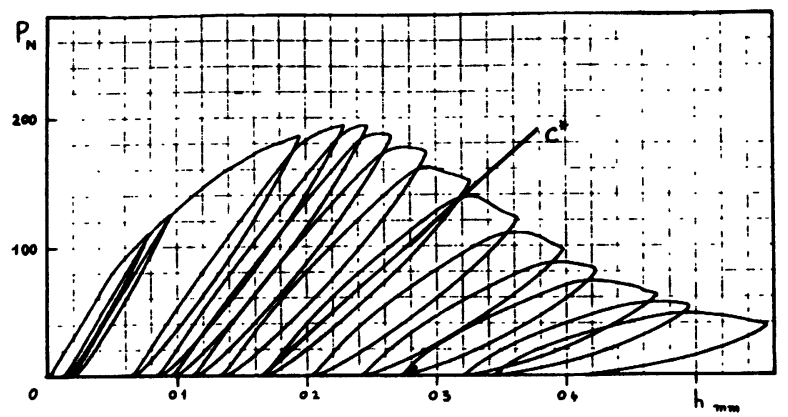

a)

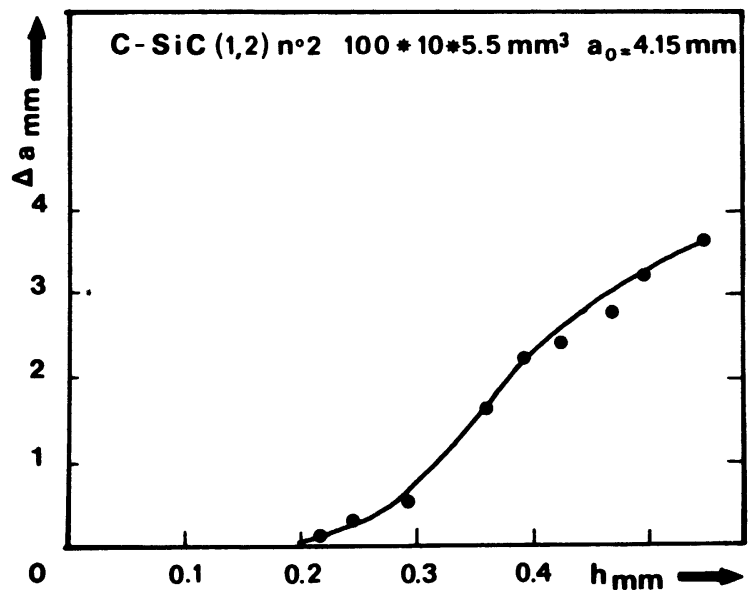

b)

Fig. 6. - Cyclage de charge d'un matériau C-SiC $(1,2)(a)$ et mesures des longueurs de fissure (b).

[Loading-unloading curve (a) and optically measured crack lengths (b) for a $\mathrm{C}$-SiC $(1,2)$ material tested in 3 point bending.] point d'appui, $P(h)$, en flexion 3 points ; on observe une petite région à comportement linéaire élastique (de 0 à 0,4 Pmax) suivie d'une large plage à comportement non linéaire non élastique avant la charge maximale. Les cycles de charge-décharge ont été réalisés pour permettre l'évaluation de la longueur de fissure au cours de l'essai. Pour cela, les compliances $C_{n}$ sont mesurées à partir des tangentes optimales aux branches de chargement (voir Fig. 6a). L'évaluation des longueurs de fissure est alors possible selon deux méthodes : celle utilisant la courbe de calibration obtenue à partir d'entailles mécaniques et celle utilisant des méthodes itératives proposées dans la littérature. Mais quelle que soit la méthode adoptée, l'estimation de la longueur de fissure au maximum de la charge conduit à une avancée $\Delta a \simeq 1$ à $2 \mathrm{~mm}$, ce qui traduit une propagation sous-critique considérable. Les longueurs de fissure observées directement en cours de rupture sur la surface polie de l'éprouvette sont également portées sur la figure $6 \mathrm{~b}$ en fonction du déplacement du point d'application de la charge. Pour chaque cycle, la longueur de fissure est mesurée pour la valeur maximale de la charge. On peut observer que l'apparition d'une fissure macroscopique, de taille suffisante pour permettre une détection au grossissement 100 , ne survient qu'au voisinage de la charge maximale. Sakai et al. [21] avaient proposé un modèle de mesure des paramètres d'énergie $\tilde{G}_{\mathrm{c}}$ et $R$, basé sur l'hypothèse d'une propagation de fissure à partir du maximum de la charge. Compte tenu de la grande déformation observée avant ce maximum de la charge, cette hypothèse semblait peu vraisemblable. Mais la corrélation du nombre total de coups $\sum N$ avec l'enregistrement $P(h)$ confirme que la fissuration (extension macroscopique de la fissure) débute au voisinage du maximum de la charge (Fig. 7). Sur la base des enregistrements acoustiques nous distinguerons l'existence de cinq domaines principaux caractérisant le chargement :

1) un faible niveau d'émission, traduisant le tassement du matériau poreux, correspond à la partie linéaire de l'enregistrement $P(h)$;

2) puis il y a accroissement de l'émission acoustique bien qu'il n'apparaisse pas de fissure macroscopique. Cette partie correspondrait à un endommagement du matériau, localisé au niveau des interfaces fibre-matrice. Ce domaine traduit le déchaussement des fibres et leur frottement dans la gaine matricielle ;

3) quand la charge maximale est atteinte, le très fort niveau d'émission acoustique correspond à l'apparition d'une fissure macroscopique ;

4) ensuite l'émission est moindre que dans le domaine précédent ; la pente $N(t)$ est comparable à celle dans le domaine 2 ; 


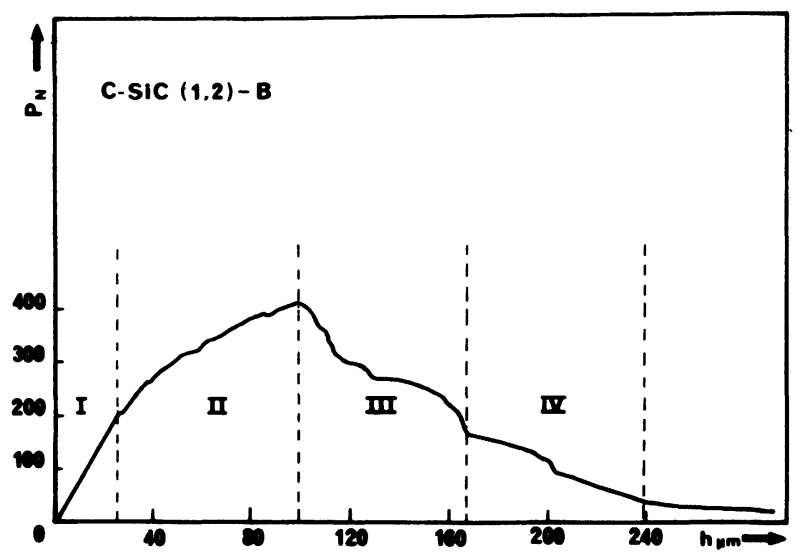

a)

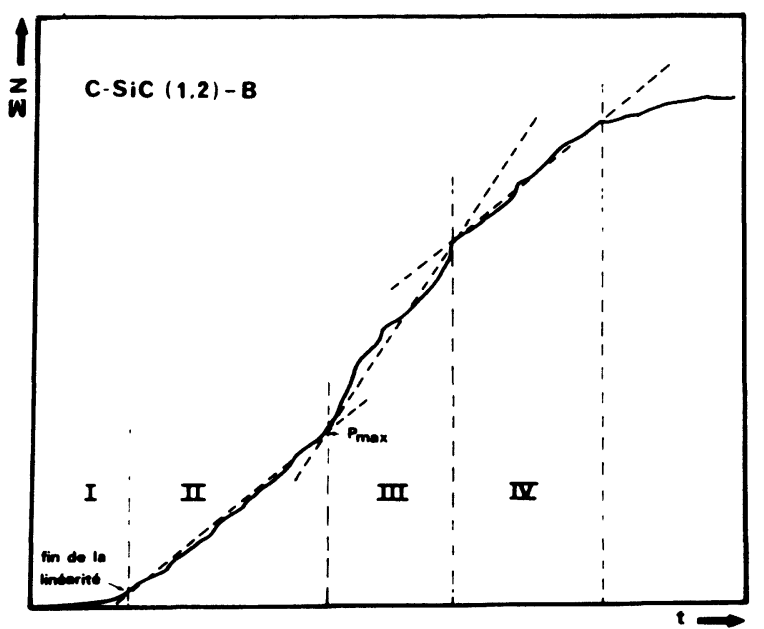

b)

Fig. 7. - Courbe de chargement monotone (a) et enregistrement acoustique associé (b) pour un matériau C-SiC $(1,2)$ testé en flexion 3 points.

[Monotonic loading curve (a) and the associated acoustic emission curve (b) for a C-SiC $(1,2)$ material tested in three point bending.]

5) ce domaine est caractérisé par un niveau constant de l'émission acoustique, la charge continuant à décroître lentement : la ruine de l'éprouvette est effective.

La fissure apparaît donc dès que la charge maximale est atteinte et, bien que sa propagation soit limitée aux domaines 3 et 4 , l'éprouvette demeure d'un seul tenant à la fin de l'essai. Etant donné que la mesure porte sur la fissuration visible de la matrice, on s'attend a priori à ce que la longueur observée soit plus petite que la longueur réelle du fait de l'effet de finesse de la fissure.

L'estimation de la longueur de fissure par la méthode itérative de Tada et al. [15] a été souvent utilisée pour le calcul des paramètres d'énergie dans le cas de matériaux composites [22, 23]. Nous avons tenu à vérifier l'applicabilité de cette relation dans le

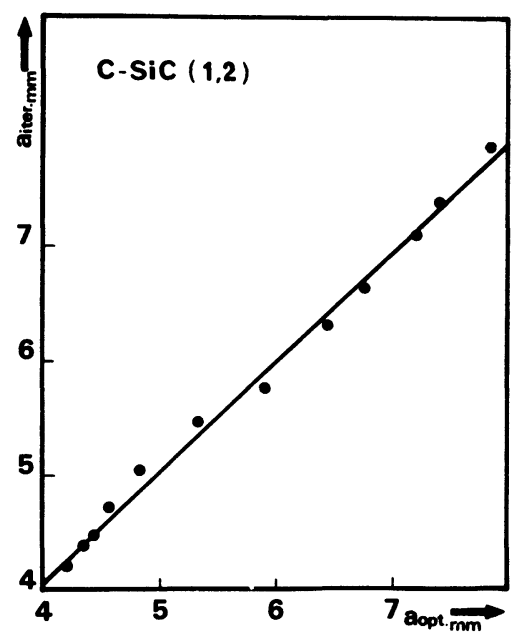

Fig. 8. - Longueurs visuelles de fissure, $a_{\mathrm{opt}}$, en fonction de celles calculées à partir de l'expression de Tada et al. [15], $a_{\text {itćr }}$.

[Optically measured crack lengths, $a_{\mathrm{opt}}$, as a function of the calculated crack length from Tada et al. [15], $a_{\text {iter }}$.

cas de ces matériaux C-SiC, avant de l'utiliser pour l'étude de l'intégrale $J$. Sur la figure 8 nous avons reporté les valeurs des longueurs de fissure mesurées visuellement en fonction de celles obtenues selon l'expression (5) :

$$
a_{n}=a_{n-1}+\frac{W-a_{n-1}}{2} \frac{C_{n}-C_{n-1}}{C_{n}} .
$$

La très bonne correspondance entre ces deux groupes de valeurs autorise à adopter l'expression (5) pour le calcul des accroissements de la longueur de fissure pour ces matériaux.

Bien que le matériau étudié ne présente pas d'effet de plasticité au sens de celui observé sur les matériaux métalliques, son comportement non linéaire non élastique est souvent abordé en terme d'intégrale $J$. L'explication réside dans l'existence d'une zone fortement perturbée et essentiellement localisée à l'avant du front de fissure, où de très forts niveaux de contrainte et de déformation sont atteints. La taille de cette zone d'endommagement avait été estimée à 0,04 inch (soit $1 \mathrm{~mm}$ ) [24] indépendamment du matériau considéré. Cependant, par comparaison avec la taille de la zone d'endommagement des matériaux céramiques denses [25], cette valeur semble être sous-estimée et elle dépendrait de la macrostructure du matériau étudié.

Les figures $9 \mathrm{a}$ et $\mathrm{b}$ présentent les courbes $J^{\mathrm{G}}, J^{\mathrm{GC}}$ et $\left(J^{\mathrm{G}}-J^{\mathrm{GC}}\right)$ en fonction de l'avancée de la fissure pour deux éprouvettes de flexion 3 points dénommées F4 et G4 (voir Tab. I). Pour les petites éprouvettes $\left(50 \times 10 \times 5 \mathrm{~mm}^{3}\right)$ les «valeurs plateau » des courbes $J^{\mathrm{G}}$ et $J^{\mathrm{GC}}$ sont respectivement 2160 $2440 \mathrm{Jm}^{-2}$ et $800-1140 \mathrm{Jm}^{-2}$; mais le niveau 


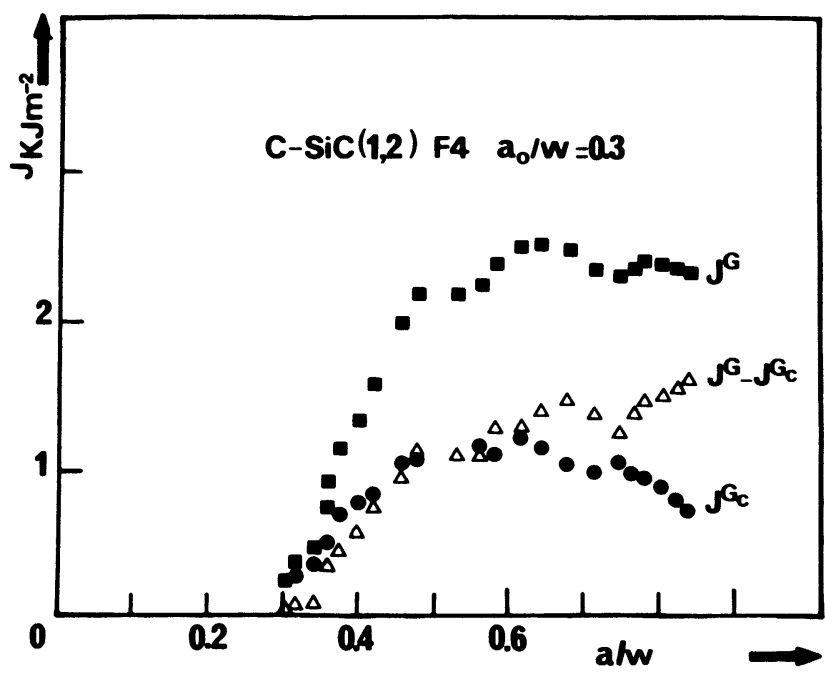

a)

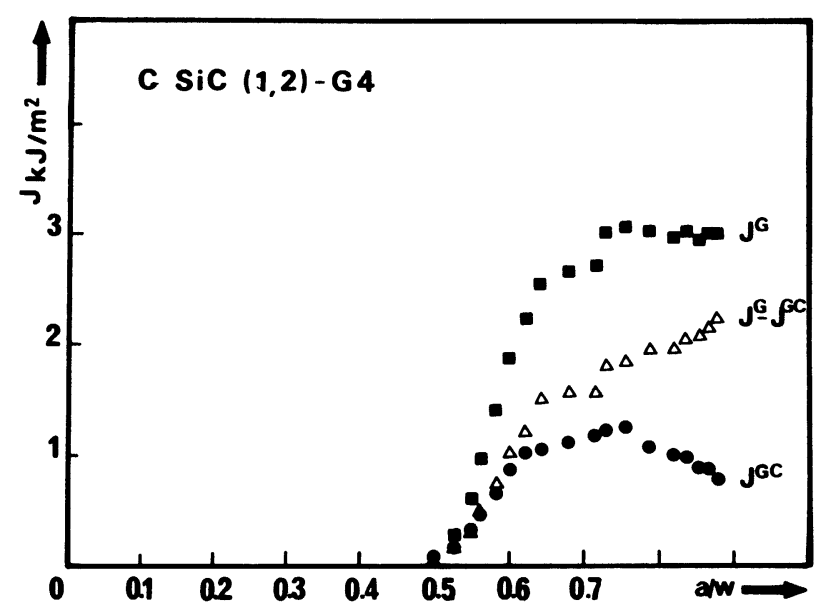

b)

Fig. 9. - Valeurs d'intégrales $J^{\mathrm{G}}, J^{\mathrm{GC}}$ en fonction de l'accroissement de la longueur de fissure : a) éprouvette $\mathrm{F}_{4}$; b) $\mathrm{G}_{4}$.

[Measured energy values $J^{\mathrm{G}}$ and $J^{\mathrm{GC}}$ as a function of the crack growth, $\Delta a$, for two specimen $F_{4}$ (a) and $G_{4}($ b).]

d'endommagement alors atteint (mesuré par l'avancée de la fissure $\Delta a=a_{\text {plateau }}-a_{0}$ ) est dépendant de la longueur de l'entaille. Une fois le plateau atteint, les valeurs $J^{\mathrm{G}}$ restent constantes alors que la courbe $J^{\mathrm{GC}}$ décroît ensuite avec l'avancée de la fissure. Ce comportement s'expliquerait par la sensibilité de la fissure à la proximité du bord supérieur de l'éprouvette. En effet la décroissance de la courbe $J^{\mathrm{GC}}$ survient quand la distance du fond de la fissure au bord de l'éprouvette est $d \simeq 2$ à $2,5 \mathrm{~mm}$ pour les lots d'éprouvettes de dimensions $50 \times 10 \times 5$ et $70 \times$ $15 \times 7,5 \mathrm{~mm}^{3}$.

Ces valeurs $d$ constituent une estimation de la taille de la zone d'endommagement, pour la famille de dimensions d'éprouvettes présentée dans cette étude. Les valeurs $d$ estimées dans le cas des éprouvettes de dimensions $100 \times 30 \times 5,5$ et $100 \times$ $18,7 \times 5,5$ sont plus élevées, sans doute du fait de la

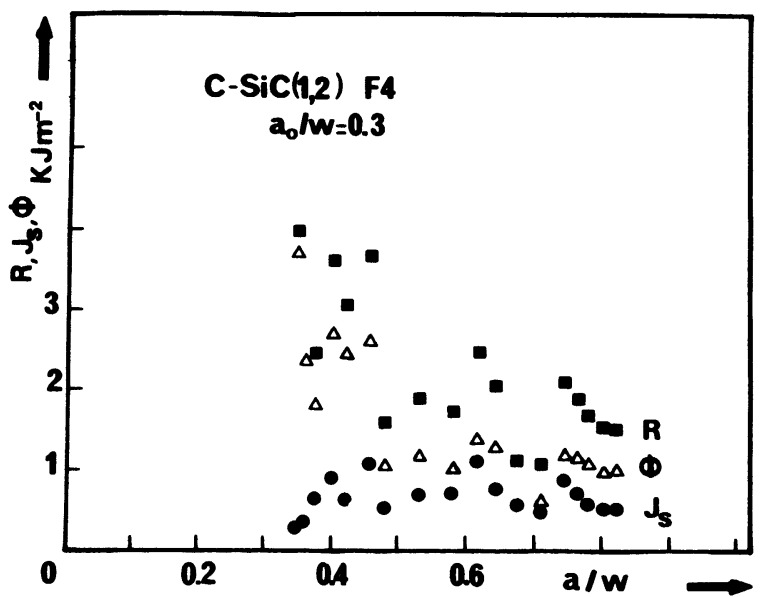

a)

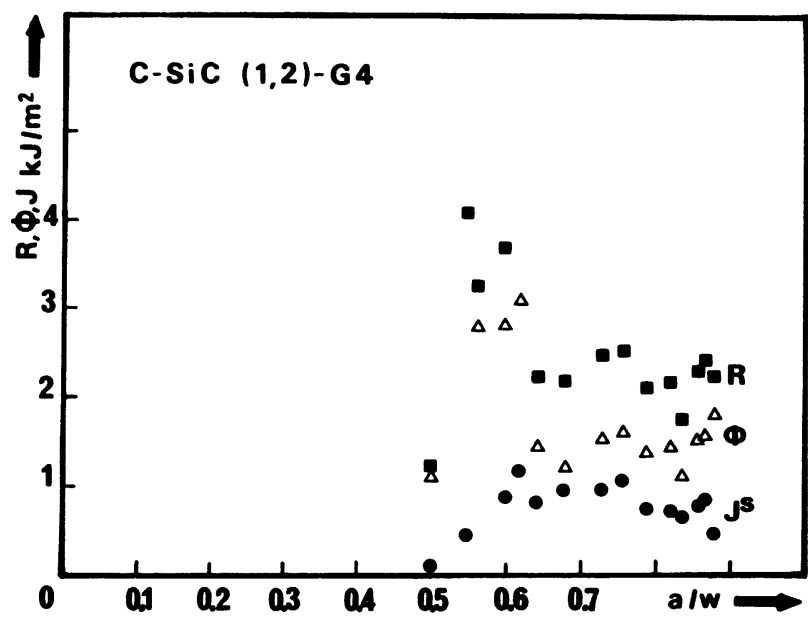

b)

Fig. 10. - Termes d'énergie $R, J^{\mathrm{S}}$ et $\phi$ en fonction de l'accroissement de la longueur de fissure pour a) éprouvette $F_{4}$; b) $G_{4}$.

[Measured energy values $R, J^{\mathrm{S}}$ and $\phi$ as a function of the crack growth, $\Delta a$, for two specimens: $F_{4}$ (a) and $\mathrm{G}_{4}$ (b).]

faible largeur de ces éprouvettes $(B=5,5 \mathrm{~mm}$ ) qui entrave le développement en volume de la zone d'endommagement.

Les valeurs $R, J^{\mathrm{S}}$ et $\phi$ mesurées par la méthode de Sakai sur les éprouvettes $F_{4}$ et $G_{4}$ sont représentées sur les figures $10 \mathrm{a}$ et $\mathrm{b}$ en fonction de l'avancée de la fissure. D'une manière générale les valeurs obtenues par la méthode de Sakai présentent plus de dispersion que celles obtenues par la méthode de Garwood ; cela serait dû à l'allure accidentée des courbes de cyclage. Les valeurs de résistance à la propagation, $R$, calculées par la méthode de Sakai sont toujours plus faibles que celles, $J^{\mathrm{G}}$, données par la méthode de Garwood; et la même relation existe entre les valeurs corrigées $J^{\mathrm{S}}$ et $J^{\mathrm{GC}}$. Cependant, pour chaque éprouvette, les valeurs respectives $R$ et $J^{\mathrm{G}}$ ou $J^{\mathrm{S}}$ et $J^{\mathrm{GC}}$ sont comparables.

Les caractéristiques dimensionnelles des éprouvet- 


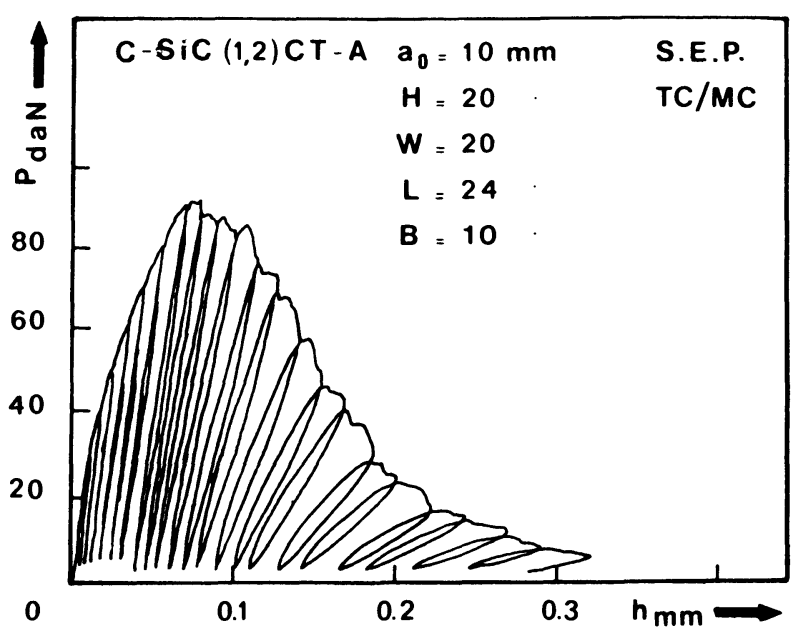

Fig. 11. - Cyclage de charge pour une éprouvette de traction compacte, C-SiC $(1,2)$.

[Loading-unloading curve for a compact tension specimen, C-SiC (1, 2).]

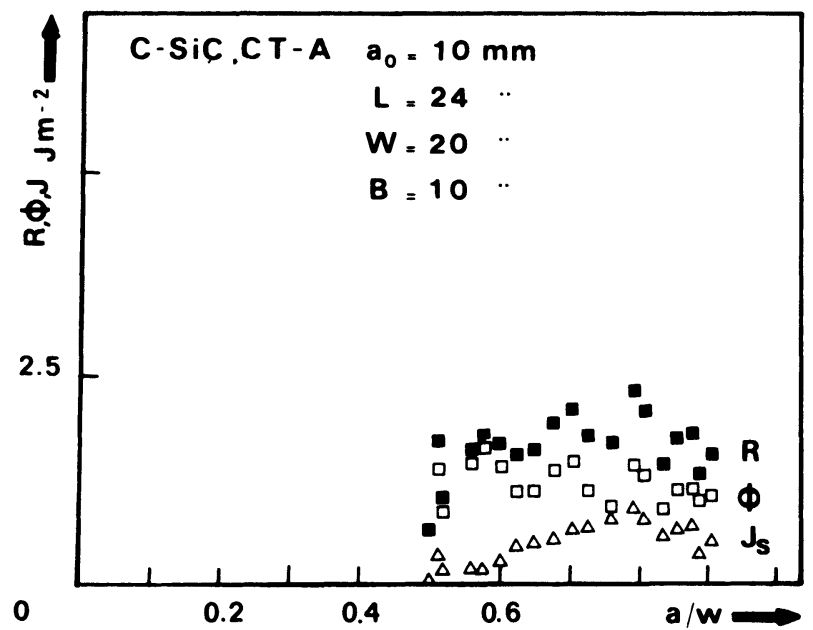

a)

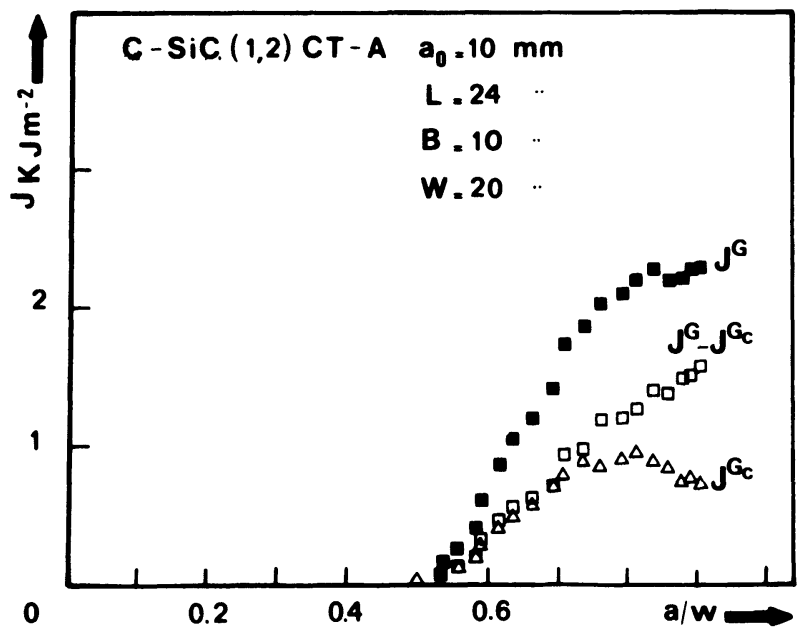

b)

Fig. 12. - Résultats obtenus sur les éprouvettes de traction compacte à partir des méthodes de Sakai (a) et Garwood (b).

[Measured energy values for a compact tension specimen using the method of Sakai (a) and the method from Garwood analysis (b).] tes étudiées et les résultats des mesures $R, J^{\mathrm{G}}, d$, $J^{S}$ et $J^{\mathrm{GC}}$ ont également été reportées dans le tableau I.

Des éprouvettes de traction compacte ont aussi été testées dans le but d'observer l'effet du type d'essai sur les résultats $R, J^{\mathrm{S}}, J^{\mathrm{G}}$ et $J^{\mathrm{GC}}$. La figure 11 présente la courbe de chargement monotone de l'éprouvette $\mathrm{CT}(\mathrm{E})$ et celle de charge-décharge de l'éprouvette CT(A): on observe que les enveloppes de ces deux enregistrements coïncident, sauf dans la première moitié avant la charge maximale où l'effet des charges-décharges abaisse le niveau du chargement. Les exploitations par la méthode de Sakai et par celle de Garwood sont indiquées sur la figure 12. La dispersion des valeurs $R_{\mathrm{c}}$ est moins prononcée que celle observée sur les essais de flexion. Les valeurs $J^{\mathrm{G}}, J^{\mathrm{GC}}, R$ et $J^{\mathrm{S}}$ sont similaires à celles obtenues sur les éprouvettes de flexion de dimension $50 \times 10 \times 5 \mathrm{~mm}^{3}$ et les valeurs $R, J^{\mathrm{S}}$, demeurent plus faibles que celles $J^{\mathrm{G}}, J^{\mathrm{GC}}$ (Tab. I).

Sur la figure 13 nous avons reporté les valeurs, $J^{\mathrm{G}}, J^{\mathrm{GC}}$, en fonction de celles correspondantes, $R$, $J^{S}$, pour toutes les éprouvettes figurant dans le tableau I. Il apparaît une relation constante entre les résultats de ces deux méthodes; la pente de la droite obtenue est 1,40 (avec $\left.\chi^{2}=0,992\right)$. Cette relation constante entre les mesures obtenues à partir de ces deux méthodes semble être due aux concepts utilisés dans les deux cas.

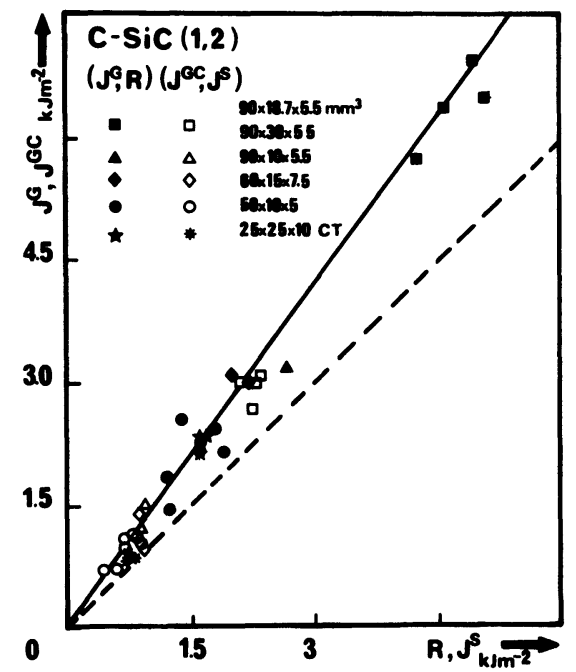

Fig. 13. - Comparaison des valeurs d'énergie obtenues par les méthodes de Garwood et Sakai, pour l'ensemble des éprouvettes testées.

[Comparison of the energy values measured using the methods of Sakai and Garwood, over all the specimens tested.]

\section{Conclusion.}

Les valeurs de l'avancée de la fissure en cours d'essai, par l'observation optique directe, sont compatibles avec celles obtenues par l'application de 
la méthode itérative de Tada et al. [15]. Les longueurs de fissure obtenues par cette méthode ont été utilisées pour évaluer les résistances à la propagation de fissures dans des composites céramiques de type C-SiC, par les méthodes d'intégrale $J$ de Garwood et al. et par la méthode de Sakai et al. Il existe une bonne corrélation entre les valeurs mesurées, par ces deux méthodes bien que les valeurs obtenues par la méthode de Sakai soient toujours plus faibles que celles obtenues par la méthode de Garwood.

On constate un effet des tailles d'éprouvettes utilisées sur les valeurs d'énergie mesurées au cours de cette étude. Cela pose le problème de l'utilisation des valeurs obtenues sur des éprouvettes de petites tailles pour le dimensionnement de structures à grande surface et de faible épaisseur. Ses mesures doivent maintenant porter sur des « plaques minces » dont les dimensions s'apparentent à celles des structures réelles faites dans ces matériaux.

D'autres mesures sont en cours pour confirmer la compatibilité des valeurs de longueurs de fissure optiques avec celles de l'expression de Tada et al. [15] et pour observer son applicabilité dans le cas de composites de type SiC-SiC.

\section{Remerciements.}

Ce travail a été fait dans le cadre du Contrat Programme Français soutenu par le CNRS, la DRET et le MRES (AIP-CNRS $n^{\circ}$ 29-85-113) et avec le soutien de la SEP que nous tenons à remercier.

\section{Bibliographie}

[1] Gerard, A., Rev. Franç. Mécanique 82 (1982) 4956.

[2] Tamuzs, V. P. et Romalis, N. B., Advanced in Fracture Research, 6th International Conference on Fracture, New-Delhi, India, Dec. 4-10 (1984) $1833-840$.

[3] Marion, R. H., ASTM STP 678 (1979) 103-111.

[4] MoussA, R., Thèse de Docteur $3^{\mathrm{e}}$ cycle, Université de Caen (1981).

[5] Steenkamp, P. A. J. M. and Hartevelt, M., Int. J. Fract. 27 (1985) R93-R98.

[6] Brown, W. F. et Srawley, J. E., ASTM STP 410.

[7] Jouin, J. M., Thèse de Docteur-Ingénieur, Université de Caen (1986).

[8] BuresCH, F. E., Fracture Mechanics of Ceramics 4 (1973) 835-847.

[9] SCHMIDT, R. A. et LuTz, T., ASTM STP 678 (1979) 166.

[10] Bouami, D., De Vadder, D., Mém. Et. Scient. Rev. Mét. 83 (1986) 249-257.

[11] Bhardwaj, M. C., Advanced Ceramic Materials 1 (1986) 311-324.

[12] LANDES, J. D. et WEI, R. P., Int. J. Fract. 9 (1973) 277-293.

[13] Trozynski, T. B. et Nicholson, P. S., J. Am. Ceram. Soc. 69 [7] (1986) C136-C137.

[14] Bornhauser, A., Kromp, K., Pabst, R. F., J. Mat. Sci. 20 (1985) 2586-2596.
[15] TADa, H., Paris, P. C. et IRwin, G. R., The stress analysis of crack handbook (Del. Research Corp., Hellertown, PA) 1973.

[16] Garwood, S. J., Robinson, J. N. et Turner, C. E., Int. J. Fract. 11 (1975) 528-530.

[17] Sakai, M., Urashima, K. et Inagaki, M., J. Am. Ceram. Soc. 66 (1983) 868-874.

[18] Dauchier, M., LamicQ, P. et Mace, J., Mém. Et. Scient. Rev. Mét. 79 (1982) 453.

[19] Gomina, M., Chermant, J. L. et Coster, M., 6th International Congress for Stereology, Gainsville, Florida USA, Oct. 10-14, 1983. Acta. Stereol. 2 Suppl. 1 (1983) 179-184.

[20] Rice, J. R., J. Appl. Mech. Trans. ASME 35 (1968) 379-386.

[21] SAKaI, M. et Bradt, R. C., Fract. Mech. Cer. Vol. 7 (1986) 127-142 Edited by R.C. Bradt.

[22] Communication INSA Lyon pour « Evaluation des Matériaux Céramiques à matrice renforcée » Marché 83 PO 522.

[23] Bouaduadja, N., Thèse de Docteur-Ingénieur, INSA Lyon (1986).

[24] Whitney, J. M., Nuismer, R. J., J. Compos. Mater. 8 (1974) 253.

[25] BuresCh, F. E., Mater. Sci. Eng. 71 (1985) 187-194. 